\title{
Discours
}

Revue de linguistique, psycholinguistique et

informatique. A journal of linguistics, psycholinguistics

and computational linguistics

1 | 2007

Varia

\section{Représentation de l'espace et du temps dans le modèle situationnel construit par un lecteur}

Point de vue psycholinguistique

\section{Yves Bestgen}

\section{CpenEdition}

\section{Journals}

Édition électronique

URL : http://journals.openedition.org/discours/100

DOI : 10.4000/discours. 100

ISSN : 1963-1723

Éditeur :

Laboratoire LATTICE, Presses universitaires de Caen

Référence électronique

Yves Bestgen, «Représentation de l'espace et du temps dans le modèle situationnel construit par un lecteur », Discours [En ligne], 1 | 2007, mis en ligne le 14 avril 2008, consulté le 19 avril 2019. URL http://journals.openedition.org/discours/100; DOI : 10.4000/discours.100

Ce document a été généré automatiquement le 19 avril 2019

\section{(c) (i) $\Theta($}

Discours est mis à disposition selon les termes de la licence Creative Commons Attribution - Pas d'Utilisation Commerciale - Pas de Modification 4.0 International. 


\section{Représentation de l'espace et du temps dans le modèle situationnel construit par un lecteur}

Point de vue psycholinguistique

Yves Bestgen

\section{Les représentations construites par le lecteur}

1 Depuis plus de vingt ans, un des défis majeurs de la psycholinguistique est de décrire la nature de la représentation mentale construite par un lecteur. Si cette question est à l'étude depuis tant d'années, c'est que, contrairement à d'autres processus comme l'accès lexical ou l'analyse syntaxique, la compréhension d'un texte est restée pendant longtemps un objet difficilement étudiable scientifiquement en raison du manque de rigueur dans sa définition et de l'absence de modèles suffisamment explicites (Rayner, Pollatsek, 1989). Tel n'est plus le cas aujourd'hui.

Comprendre un texte, c'est construire au fur et à mesure de la lecture, et donc par un processus incrémental, une représentation mentale cohérente de son contenu (Graesser, Millis, Zwaan, 1997). Cette définition présente le défaut de gommer une part importante de la complexité qui se cache derrière le terme «représentation cohérente». Plus précisément, on admet généralement que le lecteur (ou l'auditeur ${ }^{1}$ ) construit au fur et à mesure du traitement d'un texte plusieurs représentations mentales qui varient en durée de vie et en richesse (Noordman, Vonk, 1998). Il est important de noter que ces représentations mentales ne peuvent pas être observées directement. Le consensus auquel ont abouti les chercheurs doit donc être vu comme un ensemble d'hypothèses qui répondent aux contraintes qu'imposent tant la langue que l'architecture de notre système cognitif et qui rendent compte de nos comportements lors de la lecture d'un texte et de son rappel ultérieur. 


\subsection{La représentation de surface}

3 Pour présenter ces différentes représentations mentales, nous nous appuierons sur un très bref texte proposé par Garrod et Sanford (1994).

[1] Bill voulait prêter un peu d'argent à Suzanne.

Elle en avait vraiment besoin.

4 A la lecture de ces phrases, nous construisons en premier lieu une représentation mentale qui encode l'information lexicale et syntaxique du texte. Ce " code de surface " permet la conservation de la formulation exacte des phrases qui viennent d'être lues ou entendues, autorisant ainsi un retour aisé sur celles-ci en cas de difficultés de compréhension. S'agissant d'une simple copie mentale de l'information reçue, cette représentation sera différente si on change un mot ou si on déplace un complément comme dans «Bill souhaitait prêter à Suzane un peu d'argent ». De plus, elle n'encode pas les relations entre les phrases.

\subsection{La représentation propositionnelle}

5 Sauf circonstances particulières (Kintsch, Bates, 1997), le code de surface a une durée de vie très brève et est rapidement supplanté par un niveau de représentation qui reflète le sens et non la forme de surface de l'énoncé (Sachs, 1967). On s'accorde pour considérer que ce second niveau consiste en un ensemble structuré de propositions sémantiques chacune composée d'un prédicat et d'un ou plusieurs arguments. On parle d'un ensemble structuré de propositions parce qu'à ce niveau les relations entre les phrases sont encodées au travers de la prise en compte, par exemple, d'arguments communs (p. ex., Elle et Suzanne). C'est donc à ce niveau que se produit la première intégration indispensable à toute compréhension d'un texte: intégrer les mots en fonction des relations syntaxiques afin de construire les propositions sémantiques et intégrer les phrases entre elles afin de construire une représentation cohérente. Un modèle des processus cognitifs qui permettent la construction de cette représentation propositionnelle a été proposé par Kintsch et van Dijk (1978) et a été révisé ultérieurement par Kintsch (1998). Il faut toutefois noter que l'intégration interne et, tout particulièrement, l'établissement des relations entre les phrases implique presque toujours la prise en compte de connaissances préalables à la lecture du texte (Frank, Koppen, Noordman, Vonk, 2003). Pour s'en convaincre, il suffit de remplacer dans l'exemple le prénom Suzanne par Pierre et le pronom Elle par Il. Si le pronom impose toujours de relier les deux phrases, l'identification de son antécédent et donc de l'argument commun (Bill ou Pierre) ne peut se faire qu'au moyen d'une inférence qui s'appuie sur les connaissances générales $d u$ lecteur. Un troisième niveau de représentation est donc indispensable.

\subsection{La représentation situationnelle}

Cette première intégration peut être qualifiée d'interne parce qu'elle vise uniquement à relier des éléments du message entre eux. Elle est importante, mais insuffisante comme le montre la reformulation suivante du petit texte de Garrod et Sanford (1994). 
[2] Bill voulait prêter un peu d'argent à Suzanne.

Ce n'est pas agréable d'avoir de vrais copains dans le besoin.

7 Nous n'éprouvons aucune difficulté à comprendre ces deux phrases, ni à les intégrer l'une avec l'autre. Et, pourtant, aucune marque de cohésion, qu'elle soit grammaticale ou lexicale, ne nous guide. Ici, l'intégration résulte de l'injection lors des processus de compréhension de connaissances disponibles en mémoire à long terme comme, par exemple, que l'on prête plus facilement à un ami qu'à un inconnu ou encore que deux bonnes raisons pour prêter de l'argent à quelqu'un est que ce quelqu'un soit un ami et en ait besoin. Cette dernière connaissance établit une relation de conséquence-cause entre les deux phrases et donc entre les propositions sémantiques qu'elles expriment. On notera que même dans l'exemple [1], cette connexion causale doit être inférée par le lecteur sur la base de ses connaissances antérieures. Simplement, la présence d'une anaphore pronominale l'y invite, alors que dans l'exemple [2], il doit s'appuyer sur les maximes de coopération de Grice (1975) pour chercher le lien que l'auteur n'a pas signalé explicitement.

8 Les connaissances que le lecteur ajoute aux informations contenues dans le texte permettent une meilleure intégration interne, mais aussi une seconde intégration, externe celle-ci. En effet, la représentation propositionnelle se voit complétée par des connaissances. Ce que le lecteur retient du texte n'a donc plus comme seule source le texte et la représentation qui en résulte est plus riche et, également, plus variable selon le lecteur et les connaissances qu'il possédait avant la lecture. Texte et connaissances activées forment donc un troisième niveau de représentation, le plus important, classiquement appelé modèle situationnel ou modèle mental. Il s'agit d'une représentation qui va au-delà du texte de deux manières. Tout d'abord, et comme on vient de le voir, elle inclut des informations non présentes dans le texte lui-même. Ensuite, il ne s'agit pas d'une représentation du texte, mais de ce dont le texte parle comme le souligne une expression souvent citée de Glenberg, Meyer et Lindem (1987 : 70) : "Mental models represent what the text is about, not the text itself."

Ce dernier point est particulièrement explicite dans l'étude séminale de Bransford, Barclay et Franks (1972). Ces auteurs ont demandé à des personnes d'écouter des phrases comme [3a]. Chacune de ces phrases existait en deux versions, [3b] étant le seconde version de [3a]. Ultérieurement, les personnes durent décider s'ils avaient entendu très exactement des phrases comme [3a'] et [3b'], [3a'] étant la phrase test pour [3a] et [3b'] pour $[3 b]$.

[3a] Trois tortues flottaient sur un morceau de bois et un poisson nageait en dessous d'elles.

[3b] Trois tortues flottaient à côté d'un morceau de bois et un poisson nageait en dessous d'elles.

[3a'] Trois tortues flottaient sur un morceau de bois et un poisson nageait en dessous de lui.

[3b'] Trois tortues flottaient à côté d'un morceau de bois et un poisson nageait en dessous de lui.

Les résultats montrent que les participants confondent plus souvent [3a] et [3a'] que [3b] et [ $\left.3 b^{\prime}\right]$. Comment expliquer ce résultat ? Au niveau de la représentation de surface et de la représentation propositionnelle, la différence entre $3 a$ et $3 a^{\prime}$ est équivalente à celle entre $3 b$ et $3 b^{\prime}$ : «lui » a été inséré à la place de "elle ${ }^{2}$. Par contre, si on s'imagine la situation décrite par les phrases, et donc si on construit une représentation 
situationnelle, la différence entre [3b] et [3b'] est évidente (le poisson a changé de place). Par contre, [3a] et [3a'] correspondent à la même représentation situationnelle.

En conclusion, les performances des participants ne peuvent s'expliquer que si ceux-ci ont construit une représentation de la situation spatiale ${ }^{3}$ sous-jacente au texte. La même idée de représentation situationnelle s'applique à l'exemple [2]. Établir une relation causale entre les phrases permet au lecteur de comprendre la situation dont «parle » le texte. Dans le cas de narrations, le genre de textes le plus fréquemment étudié par la psycholinguistique, d'autres composantes de la situation sont aussi censées structurer le modèle situationnel comme le temps et les personnages.

\section{Conceptions théoriques expliquant la construction de la représentation situationnelle}

Que faut-il vraiment entendre par "dimensions représentées» dans le modèle situationnel? Quels impacts ces dimensions ont-elles sur les processus cognitifs qui mènent à la compréhension d'un texte ? Pour l'espace et le temps, les deux dimensions les plus étudiées ${ }^{4}$, un ensemble de recherches proposent des réponses, certes partielles, à ces questions. Mais, avant de passer en revue les connaissances disponibles à leur sujet, il est nécessaire de comprendre par quels processus cognitifs un lecteur peut construire une représentation situationnelle sur la base d'un texte. Comme on vient de le voir, cette représentation présente deux propriétés qui la distinguent des deux autres représentations construites par un lecteur : elle inclut des connaissances récupérées de la mémoire à long terme et elle représente la situation sous-jacente au texte y compris dans des aspects qui n'ont pas été explicitement énoncés (p. ex., la relation causale entre les phrases de [2]). Comme le soulignent van den Broek, Rapp et Kendeou (2005) deux grandes conceptions théoriques s'opposent à ce sujet.

\subsection{La conception mémorielle}

13 Selon la conception mémorielle de la compréhension, ces propriétés résultent de processus automatiques qui provoquent l'activation des connaissances antérieures du lecteur par un mécanisme passif (McKoon, Ratcliff, 1998; O’Brien, Rizzella, Albrecht, Halleran, 1998). Tous les concepts et les propositions qui, extraits du texte, sont insérés dans la représentation propositionnelle se signalent en parallèle à l'ensemble de la mémoire à long terme. Les informations dérivées antérieurement du texte et les connaissances générales du lecteur qui correspondent suffisamment à ces signaux sont réactivées et peuvent alors s'insérer dans le modèle situationnel. La métaphore proposée par les auteurs est celle de la résonance, issue des travaux sur la mémoire et qui donne son nom à ce modèle théorique : les connaissances compatibles avec ce qui vient d'être lu (le texte) et ce qui a déjà été compris (l'état actuel de la représentation situationnelle) entrent en résonance et deviennent donc disponibles pour prendre place dans la mise à jour du modèle situationnel. Pour essayer de rendre ce modèle plus concret, reprenons l'exemple [2]. A la lecture de la seconde phrase, le mot «ami » et l'expression «être dans le besoin » vont activer l'ensemble des connaissances du lecteur qui sont compatibles avec eux. Ils vont, si nécessaire, réactiver des éléments de la première phrase comme "argent " ou « prêter » et activer des connaissances comme celle qui veut qu'on prête plus facilement à des amis. Sur la base de l'ensemble de ces informations, la représentation situationnelle 
adéquate, c'est-à-dire incluant la relation causale entre les deux phrases, pourra être construite.

Le résultat de ces processus est donc l'activation de l'ensemble des connaissances potentiellement pertinentes pour la compréhension. Deux précisions doivent être données ici. Tout d'abord, le fait que parmi les connaissances réactivées se trouvent des informations introduites antérieurement dans le texte permet d'expliquer comment un lecteur établit la cohérence globale d'un texte. Il est d'ailleurs à noter qu'une partie importante des recherches effectuées dans le cadre de la conception mémorielle adresse tout particulièrement cette question de la réactivation de passages antérieurs du texte et néglige celle des connaissances générales comme l'ont souligné, entre autres, Lorch (1998) et Frank, Koppen, Noordman et Vonk (2005). Ensuite, il est indispensable de prévoir après le processus d'activation des connaissances, une étape de consolidation durant laquelle, seules les connaissances les plus pertinentes restent activées. En effet, le Bill des exemples [1] et [2] pourrait activer le nom de Clinton, mais il est improbable que les connaissances à propos de ce président soient utiles pour comprendre le texte. Activation, puis consolidation ou, pour reprendre la terminologie de la version la plus aboutie des modèles de la compréhension basés sur la conception mémorielle (Kintsch, 1998), construction, puis intégration, s'enchaînent et se répètent pour construire le modèle situationnel.

\subsection{La conception constructiviste}

15 La conception mémorielle n'est pas la seule théorie psycholinguistique de la compréhension de textes susceptible de rendre compte de la construction d'une représentation situationnelle. Cette conception éprouve même des difficultés à expliquer la relation spatiale au centre de l'étude de Bransford et al. (1972) qui permet au lecteur de retenir que les tortues sont au-dessus du poisson alors que le texte dit seulement que les tortues sont au-dessus du bois et que le poisson est en dessous du bois. Cette inférence ne peut pas être récupérée en mémoire à long terme ; le lecteur doit donc nécessairement passer par un processus inférentiel spécifique, basé sur la relation de transitivité (Kintsch, 1998 : 191-192). Si, par contre, le lecteur essaie de s'imaginer la situation décrite par la phrase, le problème devient très simple : la relation transitive est directement encodée dans l'image. La thèse selon laquelle le lecteur cherche activement à construire une représentation de la situation est au centre de la conception constructiviste de la compréhension (Graesser, Singer et Trabasso, 1994). Le modèle situationnel, tout particulièrement lors de la compréhension d'une narration, y est vu comme un micromonde dans lequel les personnages se meuvent, ressentent des émotions et visent des buts et où les événements s'enchaînent en fonction du temps qui passe et des relations causales qui les déterminent (Graesser, Wiemer-Hastings, Wiemer-Hastings, 2001).

La version la plus aboutie de la conception constructiviste est sans aucun doute le eventindexing model (Magliano, Zwaan, Graesser, 1999; Zwaan, Langston, Graesser, 1995) ou modèle d'indexage d'événements (Tapiero, Blanc, 2001). Celui-ci prétend que la représentation situationnelle est structurée en fonction de plusieurs dimensions comme le temps, l'espace, les relations causales. Pour suivre ces dimensions au fur et à mesure du déroulement de la narration, le lecteur construit un indice pour chacune d'elle. Sur la base du premier événement de l'histoire, il attribue à ces indices leur valeur de départ 
qu'il met ensuite à jour en fonction des événements ultérieurs. Tout au long de la lecture, l'attention du lecteur est donc focalisée sur la partie de ce micromonde qui vient d'être construite ou mise à jour. En d'autres mots, il promène une sorte de pointeur qui indique le « ici et maintenant » de l'histoire (Zwaan, Madden 2004).

\subsection{Comparaison des deux conceptions}

17 La principale différence entre la conception mémorielle et la conception constructiviste se situe au niveau du caractère automatique ou contrôlé des processus cognitifs en jeu. Pour l'event indexing model, le lecteur surveille ("monitor") les dimensions situationnelles tout au long de la lecture alors que la conception mémorielle et tout particulièrement le modèle de résonance met l'accent sur les processus, enclenchés automatiquement, qui sont censés établir, sans aucun effort du lecteur, des connexions entre le texte et les connaissances. Il ne faut cependant pas exagérer cette opposition. D'une part, la conception constructiviste admet évidemment l'existence de processus de récupération automatiques. D'autre part, la conception mémorielle doit invoquer la mise en action de processus contrôlés lorsque les processus automatiques échouent à produire une représentation cohérente du texte. Il n'en reste pas moins que l'opposition entre ces deux camps a été et est toujours particulièrement vive comme l'attestent les nombreux travaux qui confrontent les points de vue constructivistes et minimalistes ${ }^{5}$ quant à l'importance et à la priorité des processus automatiques dans la génération d'inférences. Plus généralement, les constructivistes s'intéressent tout particulièrement à la construction d'une représentation de la situation décrite par le texte, le micromonde, alors que les mémoristes insistent sur le versant «activation de connaissances en mémoire à long terme » et donc sur les processus cognitifs en jeu.

Une analyse de l'impact des informations spatiales et temporelles sur la représentation construite par le lecteur doit permettre, non seulement, de mieux comprendre les propriétés de cette représentation, mais aussi de confronter ces deux conceptions théoriques. Les deux sections suivantes résument les connaissances à propos de chacune de ces dimensions et évaluent la capacité des deux conceptions théoriques à en rendre compte.

\section{L'espace comme dimension situationnelle}

Parmi les multiples dimensions représentées dans le modèle situationnel, l'espace est certainement celle qui a reçu le plus d'attention (Bestgen, Dupont, 2003 ; Rinck, Bower, 2000). La raison de cet intérêt réside largement dans le fait qu'une situation spatiale ne peut être transmise au travers d'un texte que sous une forme linéaire, comme lorsqu'on décrit l'organisation spatiale d'une ville (Perrig, Kintsch, 1985). Pour disposer d'une représentation de la situation spatiale, le lecteur n'a d'autre choix que de la reconstruire. Il est dès lors aisé de tester les propriétés de cette représentation mentale et de déterminer si elle présente des propriétés non directement décrites dans le texte luimême (Dupont, 2001). C'est, par exemple, le cas dans les expériences de Denis et Zimmer (1992) dans lesquelles les participants étudiaient une description verbale qui localisait une série d'éléments sur deux îles. Ensuite, ils devaient indiquer si des éléments qui leur étaient présentés l'un à la suite de l'autre étaient mentionnés dans la description. Denis et Zimmer ont observé des temps de réaction plus rapides pour un élément lorsqu'il était 
précédé dans la liste par un élément qui lui était spatialement proche. Les participants ont donc été capables sur la base de la seule description verbale de construire une représentation mentale qui autorise l'activation d'éléments en fonction de leur proximité spatiale.

La situation expérimentale employée par Denis et Zimmer (1992) permet d'étudier les propriétés de la représentation situationnelle construite par le lecteur et stockée en mémoire à long terme. Par contre, elle n'autorise pas l'analyse des processus à l'oeuvre lors de la compréhension du texte. Un lecteur met-il réellement à jour sa représentation spatiale au fur et à mesure de la lecture d'un texte ? Une série d'études menées par Bower et ses collaborateurs apportent une réponse positive à cette question en montrant que lorsqu'une personne lit un récit, elle se concentre mentalement sur la position spatiale du protagoniste principal (Bower, Morrow, 1990 ; Rinck, Bower, 1995 ; Morrow, Greenspan, Bower, 1987). La procédure expérimentale est la suivante. Tout d'abord, les participants mémorisent le plan d'un bâtiment, un centre de recherche par exemple, composé de plusieurs pièces qui contiennent chacune un certain nombre d'objets comme une photocopieuse. Ensuite, les participants lisent, sur un écran d'ordinateur, des narrations qui décrivent les déplacements d'un personnage dans le bâtiment en question, ainsi que les actions qu'il entreprend. A différents moments, une tâche subsidiaire vient interrompre la lecture. Les participants voient s'afficher sur l'écran le nom de deux objets et leur tâche est d'indiquer si ces objets se trouvent dans une même pièce ou dans deux pièces différentes. Comme ils ont mémorisé auparavant le plan du bâtiment, cette tâche ne leur pose pas trop de problème. Le résultat intéressant est que la vitesse pour répondre dépend de la position du personnage selon la narration. Par exemple, lorsqu'une narration indique qu'un personnage se déplace de la cafétéria au secrétariat, les objets présents dans la pièce de départ voient leur accessibilité se réduire alors que ceux qui se trouvent dans la pièce d'arrivée deviennent plus accessibles. Il faut donc moins de temps aux participants pour répondre affirmativement à propos de paires d'objets qui se trouvent dans le secrétariat par rapport à des objets qui se trouve dans la cafétéria ou dans toute autre pièce.

21 Ainsi donc, une simple tâche de décision sur les informations apprises antérieurement est influencée par la lecture de la narration. L'explication proposée par Bower et ses collaborateurs est que le lecteur suit mentalement les déplacements du personnage durant la lecture, mettant continuellement à jour sa représentation mentale de la situation décrite dans le texte. Deux observations complémentaires viennent étayer cette thèse. Les chercheurs ont montré que les lecteurs tenaient même compte des déplacements non mentionnés explicitement dans le texte (Rinck, Bower, 1995). Par exemple, lorsque trois pièces $(\mathrm{A}, \mathrm{B}, \mathrm{C})$ se situent l'une à la suite de l'autre et que la narration indique que le personnage va de $A$ dans $C$ sans mentionner $B$, un effet de gradient d'accessibilité est observé : les objets localisés dans $C$ sont plus accessibles que ceux qui se trouvent dans B, eux-mêmes plus accessibles que ceux qui sont dans $\mathrm{A}$. De plus, l'accessibilité n'est pas seulement déterminée par la position physique du personnage, mais également pas sa position «mentale». Si le texte indique que le personnage pense à une autre pièce, c'est celle-ci qui devient la plus accessible.

22 La faiblesse principale de la situation expérimentale employée par Bower et coll. est qu'elle nécessite la mémorisation d'un plan avant la lecture. Qu'en est-il dans une situation plus habituelle dans laquelle l'espace est exclusivement présenté dans le texte? Dans une expérience devenue célèbre, Glenberg et al. (1987) ont demandé à des personnes 
de lire de petites narrations, comme celle reprise ci-dessous, présentées sur un écran d'ordinateur. A un moment donné de leur lecture, un mot était affiché et ils devaient indiquer le plus rapidement possible s'ils avaient vu ou non ce mot dans le texte qu'ils étaient en train de lire. Les textes existaient dans deux versions. Dans la première [4a], le mot cible faisait référence à un objet («survêtement ») qui restait pendant toute l'histoire près du personnage ( «il mit son survêtement »). Dans la seconde version [4b], l'objet n'était pas associé physiquement au personnage ( il enleva son survêtement »);

[4a] John se préparait pour le marathon. Après avoir fait quelques exercices d'échauffement, il mit son survêtement et commença à courir. Il parcourut sans problème la moitié du tour du lac. Un peu plus loin cependant, il ressentit une douleur musculaire.

[4b] John se préparait pour le marathon. Après avoir fait quelques exercices d'échauffement, il enleva son survêtement et commença à courir. Il parcourut sans problème la moitié du tour du lac. Un peu plus loin cependant, il ressentit une douleur musculaire.

Mot cible : survêtement

enberg et al. observe que le temps nécessaire pour indiquer que le mot cible est dans le texte est plus court pour la première version que pour la seconde. Ce résultat s'interprète de la même manière que ceux obtenus par Bower et ses collaborateurs. Le lecteur construit au fur et à mesure de la lecture une représentation de la situation décrite centrée sur le personnage principal et il consulte cette représentation pour décider si l'objet était ou non mentionné dans le texte. Dans la deuxième version, lorsque le mot cible est présenté, l'objet est très éloigné du personnage dans le modèle situationnel et le lecteur doit « d'une certaine manière » parcourir ce modèle pour le retrouver. Une série d'autres études ont confirmé cette thèse en employant d'autres mesures de l'accessibilité comme le temps nécessaire pour lire une phrase faisant référence à un élément du modèle situationnel (Levine, Klin, 2001) ou en analysant l'impact d'une incohérence situationnelle sur la compréhension (Albrecht, O’Brien, 1993; de Vega, 1995; O’Brien, Albrecht, 1992).

Il faut toutefois noter qu'une série de conditions doivent être remplies pour que le lecteur construise une représentation spatiale précise et riche de la situation décrite dans le texte. Ainsi, nombre d'effets de la dimension spatiale ne s'observent que si l'expérimentateur laisse aux participants un temps suffisant pour lire et comprendre le texte. Une condition encore plus déterminante est que la dimension spatiale soit importante pour la compréhension du texte. Pour le dire simplement, les lecteurs sont capables, s'ils doivent le faire, de construire une représentation mentale riche dans laquelle les relations spatiales entre les objets présentés dans la narration sont encodées (Bestgen, Dupont, 2003). Mais, lorsque la dimension spatiale est peu importante pour comprendre le texte ou lorsque la tâche demandée au lecteur ne nécessite pas explicitement la prise en compte des informations spatiales, celles-ci sont mal représentées, voire même non représentées dans le modèle situationnel (Levine, Klin, 2001 ; Zwaan, Radvansky, 1998 ; Radvansky, Copeland, Zwaan, 2003). La réanalyse par Jahn (2004) de la célèbre expérience de Bransford et al. (1972) est particulièrement exemplative.

Jahn (2004) a montré que les résultats rapportés par Bransford et al. (1972) n'étaient exacts que pour une partie du matériel et, ironiquement, pas pour l'exemple systématiquement cité des tortues et du morceau de bois. C'est seulement lorsque la 
situation spatiale est pertinente pour bien comprendre la phrase que les participants l'analysent et s'en souviennent ultérieurement. Le point de départ de la démonstration de Jahn est une analyse du matériel original de Bransford et al. Ce matériel contenait des scènes pacifiques comme celle des tortues, mais aussi des scènes qui mettaient en action des proies et un prédateur. Dans celles-ci, la situation spatiale est importante pour établir la dangerosité de la scène comme le montrent les exemples [5].

[5a] Deux zèbres broutaient près d'un arbuste et un lion trottinait vers lui.

[5b] Deux zèbres s'éloignaient d'un arbuste et un lion trottinait vers lui.

[5a'] Deux zèbres broutaient près d'un arbuste et un lion trottinait vers eux.

[5b'] Deux zèbres s'éloignaient d'un arbuste et un lion trottinait vers eux.

Les phrases [5a] et [5a'] décrivent une seule et même situation spatiale; Bransford et al. ont montré que les personnes ne sont pas capables de distinguer ces deux phrases. Les phrases [5b] et [5b'] décrivent deux situations spatiales différentes que, toujours selon Bransford et al., les participants peuvent distinguer. Si on remplace dans ces phrases « le lion " par "l'antilope ", on ne change rien aux situations spatiales décrites. On devrait donc obtenir les mêmes résultats. Or, ce n'est pas ce que Jahn a obtenu. Il a reproduit les résultats de Bransford et al. avec les phrases qui mettent en scène un prédateur, mais non avec celles qui décrivent des animaux pacifiques. L'explication est que, dans la condition prédateur, les phrases [5b] et [5b'] impliquent deux situations très différentes quant au risque couru par les proies. Ce n'est pas le cas lorsque le lion est remplacé par une antilope.

\section{Le temps comme dimension situationnelle}

Moins nombreuses et plus tardives que les recherches à propos de l'espace, les études à propos de la dimension temporelle n'en ont pas moins été très fructueuses. Une recherche particulièrement exemplative à ce sujet est l'adaptation en 2001 au domaine temporel de l'expérience classique de Bransford et al. (1972). Rinck, Hähnel et Becker (2001) ont construit un matériel analogue à celui de Bransford, à la seule différence que la situation décrite par les phrases était temporelle et non spatiale comme lorsqu'un pianiste et un trompettiste jouent en même temps ou l'un après l'autre et qu'une cantatrice chante avec l'un ou avec l'autre. Le reste de l'expérience était identique à celle de Bransford et les résultats furent identiques. Rinck et al. ont donc observé que les participants ne confondent pas deux situations temporelles différentes comme lorsque la cantatrice chante avec l'un ou avec l'autre, qui eux jouent l'un après l'autre. Évidemment, le matériel employé par Rinck et al. devrait être évalué d'une manière approfondie pour déterminer s'il présente des limitations similaires à celles observées par Jahn (2004) dans le matériel de Bransford et al. (1972). Pour autant qu'on puisse juger sur la base de l'exemple donné par ces auteurs, cela ne semble pas être le cas.

La majorité des autres recherches à propos de la dimension temporelle se sont intéressées à l'impact d'une rupture de la continuité temporelle sur la compréhension (Bestgen, Vonk, 1995, 2000 ; Speer, Zacks, 2005 ; Zwaan, 1996 ; Zwaan, Madden, Whitten, 2000). Tout particulièrement, Zwaan (1996) a fait lire de brèves narrations dans lesquelles une phrase cible était introduite par un adverbial temporel comme " Un instant plus tard ", " Une heure plus tard » ou "Un jour plus tard». Il a montré qu'un adverbial signalant un intervalle de temps très court facilitait l'intégration de la phrase cible avec celles qui la précédaient 
contrairement aux deux autres adverbiaux qui indiquent une rupture temporelle importante. Cet effet se marque tant au niveau de la vitesse de lecture pour la phrase cible que de l'accessibilité aux informations qui précède l'énoncé cible. Bestgen et Vonk (1995) ont montré que la simple présence d'un adverbe comme Puis au début d'une phrase suffit à produire ce type d'effet. On peut donc en conclure que le lecteur prend en compte l'évolution temporelle des événements tels que présentés dans la narration pour construire la représentation situationnelle.

Le rôle de la dimension temporelle dans la construction du modèle situationnel a aussi été mis en évidence au travers de la résolution par le lecteur d'une incohérence temporelle. Rinck et al. (2001) ont ainsi montré qu'une phrase comme "Claudia l'attendait déjà lorsqu'il descendit $d u$ train» est lue plus lentement lorsque le participant a lu quatre phrases auparavant "Le train de Markus arrivait à la gare 20 minutes avant celui de Claudia " que lorsqu'il a lu «Le train de Markus arrivait à la gare 20 minutes après celui de Claudia ». Ce ralentissement de la lecture s'observe tant chez des lecteurs qui détectent consciemment l'incohérence que chez des lecteurs qui sont incapables de la signaler malgré une interrogation spécifique à ce sujet par l'expérimentateur (Rinck et al., 2001, Exp. 4).

Comme le soulignent, entre autres, Zwaan, Madden et Stanfield (2001), l'intérêt de la dimension temporelle par rapport à la dimension spatiale est qu'elle est exprimée, en partie au moins, dans chaque phrase d'un texte au travers de nombreux moyens lexicosyntaxiques. Plusieurs recherches indiquent que les informations temporelles et aspectuelles transmises par les verbes sont prises en compte immédiatement par le lecteur et affectent la représentation situationnelle que celui-ci construit (Madden, Zwaan, 2003; Gennari, 2004; Trueswell, Tanenhaus, 1991). Magliano et Schleich (2000) ont ainsi observé que le fait qu'une action soit présentée dans une narration de manière perfective ("Stephanie changed the flat tire.») ou imperfective ("Stephanie was changing the flat tire.») affectait la vitesse avec laquelle les participants indiquaient si oui ou non l'action ("change tire ») était mentionnée dans le texte. Les actions présentées de manière imperfective restaient plus disponibles et il fallait donc moins de temps aux participants pour répondre à leur propos.

31 Il apparaît donc que les recherches à propos de la dimension temporelle sont nettement favorables aux conceptions constructivistes. Toutefois, comme pour la dimension spatiale, quelques études remettent en cause cette thèse. Zwaan, Madden et Whitten (2000) ont nuancé les conclusions de Zwaan (1996) en montrant que l'effet d'un adverbial temporel comme « Une heure plus tard » n'est pas lié à la rupture temporelle qu'il induit, mais au fait que sa longueur est suffisante pour indiquer que la situation (événement ou activité) préalablement en cours est terminée ou non. En d'autres mots, la simple présence d'un adverbial signalant une rupture temporelle importante est insuffisante pour produire une mise à jour du modèle situationnel et le ralentissement de la lecture que cette mise à jour induit. Bestgen et Vonk (2000) ont montré qu'une expression comme "Vers quatre heures" introduite au début d'une phrase qui est en discontinuité thématique avec celles qui la précèdent facilite le traitement du changement de thème. Il est donc incorrect de penser que tout adverbial temporel dans toute narration produit nécessairement un ralentissement de la lecture. Radvansky, Zwaan, Curiel et Copeland (2001) n'ont pas trouvé d'effet sur les temps de lecture de la présence de ruptures temporelles dans des textes historiques. 


\section{Discussion générale}

\subsection{Comparaison des effets des dimensions spatiales et temporelles} suffisent pas à remettre en cause les études qui confrontent directement l'impact des différentes formes de ruptures situationnelles sur la compréhension d'un texte et qui plaident, systématiquement, en faveur d'une prépondérance de la dimension temporelle (Radvansky et al., 2001 ; Zwaan, Magliano, Graesser, 1995). Ces recherches emploient une méthodologie corrélationnelle basée sur l'analyse des variables qui prédisent les temps de lecture. On procède en demandant à des personnes de lire des textes relativement longs et en enregistrant les temps de lecture pour chaque phrase. Parallèlement, les textes sont analysés linguistiquement afin de répertorier toute une série de facteurs susceptibles d'influencer les temps de lecture comme la longueur des phrases ou le nombre d'arguments nouveaux dans les propositions sémantiques qui composent les phrases. On note également la présence d'une discontinuité temporelle, spatiale ou causale entre chaque phrase et celles qui la précèdent. L'analyse vise à déterminer dans quelle mesure chacun de ces facteurs prédit les temps de lecture. On observe systématiquement, et logiquement, que la longueur des phrases est un excellent prédicteur. Un résultat beaucoup plus intéressant est que la présence de ruptures situationnelles affecte aussi les temps de lecture. Ce genre d'études permet donc de classer les différentes dimensions situationnelles en fonction de leur impact respectif sur la vitesse de lecture. Cette comparaison montre que les discontinuités temporelles ont beaucoup plus d'effet sur la vitesse de lecture que les discontinuités spatiales. Bien plus, dans la majorité des expériences, les ruptures spatiales n'ont tout simplement pas d'effet sur la vitesse de lecture. Ce résultat a été reproduit récemment par Therriault, Rinck et Zwaan (2006) dans une étude qui combinait la méthode qui vient d'être décrite à une situation expérimentale plus contrôlée (voir aussi Rinck, Weber, 2003). Même s'il faut noter que ces 
recherches portent sur les processus à l'oeuvre durant la lecture et non sur les propriétés de la représentation situationnelle construite par le lecteur, on aboutit à la conclusion que la dimension temporelle affecte plus nettement la construction de la représentation situationnelle que ne le fait la dimension spatiale.

Comment expliquer cette prépondérance de la dimension temporelle ? En premier lieu, le temps est une information omniprésente dans un texte. Il apparaît d'une manière ou d'une autre dans chaque phrase (Zwaan et al., 2001). L'auteur d'un texte n'ayant d'autre choix que de donner de nombreuses indications temporelles, il semble logique que ces mêmes informations affectent profondément la compréhension et la représentation construite lorsqu'on comprend un texte. Ensuite, plusieurs chercheurs ont fait remarquer que, comparé à l'espace, le temps est nettement plus lié à la dimension causale, par le simple fait qu'une cause doit se produire avant sa conséquence (Rinck et al., 2001). En d'autres mots, le temps et l'espace n'auraient d'impact sur la construction de la représentation situationnelle que lorsqu'ils mettent en jeu une relation causale et dans ce genre de situation, l'espace aurait autant d'impact que le temps. Simplement, dans une narration, le temps serait plus souvent à la base d'une relation causale. Ce dernier point est très important. Il suggère que ce qui est fondamental pour le lecteur n'est pas tant de suivre les dimensions situationnelles que de comprendre l'histoire, un point au centre de la conception constructiviste, mais également compatible avec la conception mémorielle comme nous allons le voir.

\subsection{Espace, temps, et conceptions théoriques}

36 Les travaux résumés ci-dessus s'accordent bien avec la conception constructiviste. On ne s'en étonnera pas trop étant donné que la plus grande partie de ces études ont été effectuées par des chercheurs à l'origine de l'event-indexing model. Ce modèle présente également l'avantage de pouvoir s'adapter aisément aux résultats expérimentaux puisque le nombre de dimensions suivies par le lecteur n'est pas précisément défini (Tapiero, Blanc, 2001). De plus, ce modèle prévoit explicitement que certaines dimensions peuvent être surveillées par le lecteur avec plus ou moins d'attention (Zwaan, Radwansky, 1998). Cette liberté laissée au lecteur permet de rendre compte aisément des résultats qui soulignent la prépondérance de la dimension temporelle sur la dimension spatiale. Enfin, ce modèle souligne que la nature de la tâche demandée aux lecteurs affecte profondément la construction du modèle situationnel.

Les travaux qui montrent que le lecteur suit les dimensions situationnelles durant sa lecture sont nettement moins compatibles avec la conception mémorielle. Par contre, celle-ci s'accorde aisément avec les observations qui montrent qu'une série de conditions doivent être remplies pour espérer mettre en évidence une éventuelle surveillance par le lecteur de ces dimensions. De plus, usant de la même adaptabilité que l'event-indexing model, cette conception soutient que lorsque la simple activation automatique et passive de connaissances ne suffit pas à comprendre le texte, le lecteur enclenche des processus contrôlés tels que ceux décrits par la conception constructiviste.

38 La confrontation de ces deux conceptions théoriques aux résultats des expériences impose donc de proposer une conclusion toute en nuance. Il est bien établi que le lecteur ou l'auditeur est capable de suivre les dimensions situationnelles et qu'il le fera si les objectifs de sa lecture le lui imposent. Si vous écoutez une personne qui décrit au téléphone l'agencement de l'appartement que vous venez de louer pour les vacances, 
vous pourrez vous en construire une représentation spatiale, au prix de quelques efforts cognitifs sans doute. Mais, par contre, si vous êtes passionné par l'intrigue policière d'un roman, il y a peu de chance que la même description d'un appartement, a priori sans lien avec l'intrigue, vous conduise à construire une représentation spatiale particulièrement riche. Selon la situation de lecture et les objectifs du lecteur donc, la représentation situationnelle construite sera très différente. Dans certains cas, il est probable que le lecteur surveillera attentivement les dimensions situationnelles. Dans d'autres, la représentation situationnelle résultera presque exclusivement de l'activation de connaissances par des mécanismes automatiques. Si on prend également en compte le fait que l'event-indexing model n'explique pas par quels processus les connaissances nécessaires à la construction du micromonde sont récupérées de la mémoire à long terme, on arrive à la conclusion que seul un modèle combinant la conception mémorielle et la conception constructiviste est viable (Noordman, Vonk, 1998 ; van den Broek et al., 2005).

Une conception de ce type a été proposée par Gernsbacher $(1990,1997)$ sous le nom de " structure building framework ». Gernsbacher conceptualise la compréhension comme une oscillation entre deux étapes : le développement d'une structure en cours de construction et le passage à une nouvelle structure. Les premiers mots d'un texte sont utilisés pour construire la première structure. Celle-ci est étendue par adjonction des informations nouvelles tant que ces dernières sont cohérentes avec celles qui précèdent. Cette cohérence se marque dans la continuité spatiale, temporelle, causale, ... Un énoncé qui apporte de nouvelles informations à propos de ce qui s'est passé au même endroit, dans la même période de temps et qui est lié causalement à ce qui précède est aisément intégré dans la structure en cours de développement. Par contre, lorsqu'il y a une rupture de cette cohérence, le lecteur doit construire une nouvelle structure, étape qui consomme des ressources cognitives supplémentaires. C'est le cas lorsqu'il y a un changement de lieu, de temps ou une absence de relation causale.

Une thèse similaire est sous-jacente aux analyses de Charolles (1997) et Charolles, Le Draoulec, Pery-Woodley et Sarda (2005) à propos des expressions qui introduisent les cadres du discours. Il s'agit d'adverbiaux extra-prédicatifs antéposés qui indexent les informations qu'ils préfixent en fonction d'un critère qui peut, entre autres, être temporel (En 2004) ou spatial (En France). La portée de ces adverbiaux peut s'étendre audelà de la phrase et donc gouverner l'interprétation d'un segment de texte. Ces cadres contribuent à subdiviser et à répartir les informations apportées par le discours au fur et à mesure de son développement. Ils participent de la sorte à son organisation. Ils favorisent également la compréhension du texte en gérant les opérations de mobilisation des connaissances requises pour l'interprétation des relations entre propositions.

Dans ce cadre qui vient d'être tracé, les signaux linguistiques de la continuité ou de la discontinuité spatiale et temporelle n'interviennent pas uniquement lorsque les objectifs de lecture imposent au lecteur de surveiller ces dimensions et de construire une représentation en fonction de celles-ci. En effet, ces signaux ont un impact sur la compréhension dès qu'ils posent un problème pour l'intégration interne du texte ou dès qu'ils imposent la mobilisation de nouvelles connaissances.

Cette analyse conduit à trois conséquences qui sont autant de pistes pour de futures recherches. Tout d'abord, l'espace et le temps n'ont pas, jusqu'à présent, été traités d'une manière équitable quant au rôle de ces signaux. Comme on l'a vu, l'étude du temps s'est focalisée principalement sur les moyens linguistiques permettant d'exprimer celui-ci dans les textes (p. ex. les adverbiaux temporels) et sur leur impact lors de la 
compréhension. Par contre, la manière dont les informations spatiales sont présentées dans un texte a retenu bien peu l'attention au profit de la question de savoir si et comment la multidimensionnalité de l'espace peut être reconstruite par le lecteur sur la base de l'organisation linéaire d'un texte (voir, par exemple, les expériences de Perrig et Kintsch (1985) sur la compréhension par le lecteur d'une description d'une ville et la capacité à inférer des relations spatiales). Il serait donc utile de rediriger les recherches à propos de la dimension spatiale afin de déterminer si des adverbiaux spatiaux, insérés en début de phrases, ont un impact similaire à celui des adverbiaux temporels comme le suggèrent plusieurs auteurs (Bestgen, Costermans, 1994; Charolles, 1997; Virtanen, 1992).

Une seconde conséquence mène à envisager l'impact d'autres dimensions "situationnelles" comme la dimension énonciative (Selon l'ambassadeur) également exprimée dans les textes par des adverbiaux extra-prédicatifs antéposés (Charolles et al., 2005). Cela imposerait évidemment d'étudier d'autres types de textes, un élargissement qui viendrait bien à propos étant donné que l'event-indexing model rencontre des problèmes lorsqu'il est appliqué aux textes historiques (Radvansky et al., 2001).

Enfin, une étude de ces signaux linguistiques permettrait de mieux confronter les deux conceptions théoriques. En effet, les mémoristes ne prévoient pas de traitements spécifiques pour ces signaux alors que les constructivistes leur accordent un rôle très important (Magliano, Schleich, 2000). Pour ce qui concerne la conception mémorielle, on peut penser que ces signaux pourraient avoir deux effets. Tout d'abord, et comme on l'a $\mathrm{vu}$, ils imposent un traitement actif lorsqu'ils introduisent une rupture trop forte de la cohérence locale. Le deuxième effet, plus intéressant, devrait s'observer lorsque ces signaux, et plus particulièrement les expressions adverbiales insérées en début de phrase, entrainent un changement important au niveau des connaissances récupérées en mémoire à long terme par le mécanisme de résonance. Une telle situation pourrait se produire lorsqu'une expression adverbiale comme "A la maison» est insérée dans une histoire qui se déroule jusque-là dans un parc d'attractions.

\section{Conclusion}

L'étude des dimensions spatiales et temporelles s'est largement développée durant les quinze dernières années. Si des modèles théoriques ont été proposés et testés, il n'en reste pas moins que ce champ de recherche présente encore trois faiblesses principales auxquelles il faudra remédier. Tout d'abord, comme on vient de le voir, une analyse fine des dispositifs linguistiques qui matérialisent les dimensions spatiale et temporelle dans les textes est indispensable. Ensuite, la critique déjà formulée en 1997 par Lorch et van den Broek à l'encontre d'une psycholinguistique textuelle uniquement consacrée à l'étude des narrations est hélas toujours d'actualité. Tester les prédictions de modèles théoriques lors de la compréhension de textes de différents genres est donc de plus en plus nécessaire. Enfin, une analyse théorique de la notion d'objectifs de lecture est indispensable puisque, comme on l'a vu, ces objectifs déterminent la nature des processus cognitifs mis en place par le lecteur et les facteurs qui affectent la construction de la représentation situationnelle.

Cette recherche a bénéficié du soutien de l'Agence nationale de la Recherche (Projet "Spatial Framing Adverbials: Linguistic and psycholinguistic approaches") et du Gouvernement de la 
Communauté française de Belgique ("Action de Recherche concertée »). L'auteur souhaite remercier les experts pour leurs nombreux commentaires très précieux.

\section{BIBLIOGRAPHIE}

ALBRECHT, J.E., O'BRIEN, E.J., 1993. Updating a mental model: maintaining both local and global coherence. Journal of Experimental Psychology : Learning, Memory and Cognition 19 : 1061-1070.

BESTGEN, Y., COSTERMANS, J., 1994. Time, space, and action : exploring the narrative structure and its linguistic marking. Discourse Processes 17 : 421-446.

BESTGEN, Y., DUPONT, V., 2003. The construction of spatial situation models during reading. Psychological Research 67 : 209-218.

BESTGEN, Y., VONK, W., 1995. The role of temporal segmentation markers in discourse processing. Discourse Processes 19 : 385-406.

BESTGEN, Y., VONK, W., 2000. Temporal adverbials as segmentation markers in discourse comprehension. Journal of Memory and Language $42: 74-87$.

BOWER, G.H., MORROW, D.G., 1990. Mental models in narrative comprehension. Science 247 : 44-48.

BRANSFORD, J.D., BARCLAY, J.R., FRANCKS, J.J., 1972. Sentence memory : a constructive versus interpretive approach. Cognitive Psychology 3 : 193-209.

CHAROLLES, M., 1997. L'encadrement du discours - univers, champs, domaines et espaces. Cahier de Recherche Linguistique $6: 1-73$.

CHAROLLES, M., LE DRAOULEC, A., PERY-WOODLEY, M-P., SARDA, L., 2005. Temporal and spatial dimensions of discourse organization. French Language Studies $15: 115-130$.

de VEGA, M., 1995. Backward updating of mental models during continuous reading of narratives. Journal of Experimental Psychology : Learning, Memory and Cognition 21 :373-385.

DENIS, M., ZIMMER, H.D., 1992. Analog properties of cognitive maps constructed from verbal descriptions. Psychological Research, Psychologische Forschung 54 : 286-298.

DUPONT, V., 2001. Étude des représentations mentales construites lors de la compréhension de textes procéduraux. Thèse de Doctorat non publiée. Faculté de Psychologie, Université catholique de Louvain, Louvain-la-Neuve, Belgique.

FRANK, S.L., KOPPEN, M., NOORDMAN, L.G.M., VONK, W., 2003. Modeling knowledge-based inferences in story comprehension. Cognitive Science $27: 875-910$.

FRANK, S.L., KOPPEN, M., NOORDMAN, L.G.M, VONK, W., 2005. Modeling Multiple Levels of Text Representation. In F. SCHMALHOFER, C.A. PERFETTI (eds.), Higher level language processes in the brain: inference and comprehension processes. Mahwah, NJ: Erlbaum. 133-157.

GARROD, S.C., SANFORD, A.J., 1994. Resolving sentences in a discourse context : how discourse representation affects language understanding. M.A. GERNSBACHER (ed.), Handbook of Psycholinguistics. San Diego : Academic Press. 675-698. 
GENNARI, S.P., 2004. Temporal references and temporal relations in sentence comprehension. Journal of Experimental Psychology :Learning, Memory and Cognition 30 : 877-890.

GERNSBACHER, M.A., 1990. Language comprehension as structure building. Hillsdale : LEA.

GERNSBACHER, M.A., 1997. Coherence cues mapping during comprehension. J. COSTERMANS, M. FAYOL (eds.) Processing interclausal relationships in the production and comprehension of text. Erlbaum : Hillsdale. 3-21.

GLENBERG, A.M., MEYER, M., LINDEM, K., 1987. Mental models contribute to foregrounding during text comprehension. Journal of Memory and Language $26: 69-83$.

GRAESSER, A.C., MILLIS, K.K., ZWAAN, R.A., 1997. Discourse comprehension. Annual Review of Psychology $48: 163-189$

GRAESSER, A.C., SINGER, M., TRABASSO, T., 1994. Constructing inferences during narrative text comprehension. Psychological Review 101 : 371-395.

GRAESSER, A.C., WIEMER-HASTINGS, P., WIEMER-HASTINGS, K., 2001. Constructing Inferences and Relations during Text Comprehension. T. SANDERS, J. SCHILPEROORD, W. SPOOREN (eds.) Text representation : linguistic and psycholinguistic aspects. Benjamins : Amsterdam/Philadelphia. 249-271.

GRICE, H.P., 1975/1979. Logique et conversation. Communications $30:$ 57-72.

JAHN, G., 2004. Three turtles in danger : spontaneous construction of causally relevant spatial situation models. Journal of Experimental Psychology : Learning, Memory and Cognition 30 : 969-987. KINTSCH, W., 1998. Comprehension : A paradigm for cognition. CUP, Cambridge.

KINTSCH, W., BATES, E., 1977. Recognition memory for statements from a classroom lecture. Journal of Experimental Psychology : Human Learning and Memory 3 : 150-159.

KINTSCH, W., VAN DIJK, T.A., 1978. Toward a model of text comprehension and production. Psychological Review 85 : 363-394.

LEVINE, W.H., KLIN, C.M., 2001. Tracking of spatial information in narratives. Memory and Cognition $29: 327-335$

LORCH, R.F. Jr, 1998. Memory-based text processing: Assumptions and issues. Discourse Processes 26 : 213-221.

LORCH, R.F.JR, VAN DEN BROEK, P., 1997. Understanding reading comprehension: Current and future contributions of cognitive science. Contemporary Educational Psychology 22 : 213-246.

MADDEN, C.J., ZWAAN, R.A., 2003. How does verb aspect constrain event representations? Memory and Cognition $31: 663-672$

MAGLIANO, J.P., ZWAAN, R.A., GRAESSER, A.C., 1999. The role of situational continuity in narrative understanding. In H.E. van OOSTENDORP, S.R. GOLDMAN (eds.), The construction of mental representations during reading. Mahwah, NJ: Erlbaum. 219-245.

MAGLIANO, J.P., SCHLEICH, M.C., 2000. Verb aspect and situation models. Discourse Processes 29 : 83-112. MCKOON, G., RATCLIFF, R., 1992. Inference during reading. Psychological Review 99 : 440-466.

MCKOON, G., RATCLIFF, R., 1995. The minimalist hypothesis : directions for research. C.A. WEAVER, S. MANNES, C.R. FLETCHER (eds.). Discourse Comprehension. Hilsdall LEA. 97-116.

MCKOON, G., RATCLIFF, R., 1998. Memory-based language processing : psycholinguistic research in the 1990s. Annual Review of Psychology, $49: 25-42$. 
MORROW, D.G., GREENSPAN, S., BOWER, G.H., 1987. Accessibility and situation models in narrative comprehension. Journal of Memory and Language 26 : 165-187.

NOORDMAN, L.G.M., VONK, W., 1998a. Discourse comprehension. In A.D. FRIEDERICI (ed.), Language comprehension : a biological perspective. Springer : Berlin. 229-262.

NOORDMAN, L.G.M., VONK, W., 1998b. Memory-based processing in understanding causal information. Discourse Processes 28 : 191-221.

O'BRIEN, E.J., ALBRECHT, J.E., 1992. Comprehension strategies in the development of a mental model.

Journal of Experimental Psychology : Learning, Memory and Cognition 18 : 777-784.

O’BRIEN, E.J., RIZZELLA, M.L., ALBRECHT, J.E., HALLERAN, J.G., 1998. Updating a situation model : A memory-based text processing view. Journal of Experimental Psychology: Learning, Memory and Cognition $24: 1200-1210$.

PERRIG, W., KINTSCH, W., 1985. Propositional and situational representations of text. Journal of Memory and Language 24 : 503-518.

RADVANSKY, G.A., COPELAND, D.E., ZWAAN, R. A., 2003. Aging and functional spatial relations in comprehension and memory. Psychology and Aging 18 : 161-165.

RADVANSKY, G.A., ZWAAN, R.A., CURIEL, J.M., COPELAND, D.E., 2001. Situation model and aging. Psychology and Aging $16: 145-160$.

RAYNER, K., POLLATSEK, A., 1989. The Psychology of Reading. Englewood Cliffs: Prentice Hall.

RINCK, M., BOWER, G.H., 1995. Anaphora resolution and the focus of attention in situation models. Journal of Memory and Language 34 : 110-131.

RINCK, M., BOWER, G., 2000. Temporal and spatial distance in situation models. Memory and Cognition $28: 1310-1320$.

RINCK, M., HÄHNEL, A., BECKER, G., 2001. Using temporal information to construct, update, and retrieve situation models of narratives. Journal of Experimental Psychology : Learning, Memory and Cognition $27: 67-80$.

RINCK, M., WEBER, U., 2003. Who when where : an experimental test of the event-indexing model. Memory and Cognition $31: 1284-1292$.

SACHS, J. S., 1967. Recognition memory for syntactic and semantic aspects of connected discourse, Perception and Psychophysics 2 : 437-442.

SPEER, N.K., ZACKS, J.M., 2005. Temporal changes as event boundaries : processing and memory consequences of narrative time shifts. Journal of Memory and Language 53 : 125-140.

TAPIERO, I., BLANC, N., 2001. Vers la prise en compte de la caractéristique multidimensionnelle des représentations mentales construites à partir des textes narratifs : apports théoriques, empiriques et questions. Année Psychologique 101 : 655-682.

THERRIAULT, D.J., RINCK, M., ZWAAN, R.A., 2006. Assessing the influence of dimensional focus during situation model construction. Memory and Cognition 34 : 78-89.

TRUESWELL, J.C., TANENHAUS, M.K., 1991. Tense, temporal context, and syntactic ambiguity resolution. Language and Cognitive Processes 6 :303-338.

VAN DEN BROEK, P., RAPP, D.N., KENDEOU, P., 2005. Integrating memory-based and constructionist processes in accounts of reading comprehension. Discourse Processes 39 : 299-316. 
VIRTANEN, T., 1992. Discourse functions of adverbial placement in English, Åbo: Åbo Akademi University Press.

ZWAAN, R.A., 1996. Processing narrative time shifts. Journal of Experimental Psychology : Learning, Memory and Cognition $22: 1196-1207$.

ZWAAN, R.A., Langston, M.C., Graesser, A.C., 1995. The construction of situation models in narrative comprehension: An event-indexing model. Psychological Science 6 : 292-297.

ZWAAN, R.A., \& MADDEN, C.J., 2004. Updating situation models. Journal of Experimental Psychology:

Learning, Memory and Cognition $30: 283-288$.

ZWAAN, R.A., MADDEN, C.J., STANFIELD, R.A., 2001. Time in narrative comprehension : a cognitive

perspective. In D. SCHRAM, G. J. STEEN (eds.), The Psychology and Sociology of Literature. John

Benjamins, Amsterdam. 71-86.

ZWAAN, R.A., MADDEN, C.J. WHITTEN, S.N., 2000. The presence of an event in the narrated situation affects its availability to the comprehender. Memory and Cognition $28: 1022-1028$.

ZWAAN, R. A., MAGLIANO, J. P., GRAESSER, A. C., 1995. Dimensions of situation model construction in narrative comprehension. Journal of Experimental Psychology : Learning, Memory and Cognition 21 : 386-397.

ZWAAN, R.A., RADVANSKY, G.A., 1998. Situation models in language comprehension and memory. Psychological Bulletin 123 : 162-185.

\section{NOTES}

1. Comme la majorité des études discutées ici ont eu recours à la modalité écrite, on fera le plus souvent référence dans la suite à cette seule modalité même si tout laisse penser que les conclusions auxquelles on aboutit sont également pertinentes pour la modalité orale.

2. La différence entre les phrases [3a] et [3b] (sur versus à côté de) n'est pas pertinente pour distinguer entre elles les versions [3a] et [3a'], ni les versions [3b] et [3b'].

3. On verra plus loin que cette interprétation classique doit être nuancée.

4. Pour une discussion des autres dimensions censées être représentées dans le modèle situationnel voir par exemple Tapiero et Blanc (2001) et Zwaan et Radvansky (1998). On notera que celles-ci sont autant de réminiscences des unités de la tragédie classique, auquel s'ajoute, par défaut, l'unité d'acteur (Bestgen et Costermans, 1994).

5. Minimaliste est le nom donné par McKoon et Ratcliff $(1992,1995)$ à l'hypothèse, sous-jacente à la conception mémorielle. selon laquelle les seules inférences couramment générées par un lecteur sont basées sur des connaissances hautement disponibles ou sont nécessaire pour établir la cohérence locale d'un texte.

\section{RÉSUMÉS}

Depuis plus de vingt ans, un des défis majeurs de la psycholinguistique est de décrire la nature de la représentation mentale construite par un lecteur. Si cette question est à l'étude depuis tant 
d'années, c'est que, contrairement à d'autres processus comme l'accès lexical ou l'analyse syntaxique, la compréhension d'un texte est restée pendant longtemps un objet difficilement étudiable scientifiquement en raison du manque de rigueur dans sa définition et de l'absence de modèles suffisamment explicites. Tel n'est plus le cas aujourd'hui. Les chercheurs s'accordent, en effet, pourdéfinir la compréhension d'un texte comme la construction d'une représentation situationnelle qui combine les propositions extraites du texte à celles qui ont été réactivées en mémoire à long terme afin de constituer une représentation non du texte, mais de ce dont le texte parle. Parmi les multiples dimensions censées être représentées dans le modèle situationnel, l'espace et le temps sont certainement ceux qui ont retenu le plus d'attention. L'objet du présent travail est de confronter nos connaissances à propos du rôle de ceux-ci lors de la construction du modèle situationnel. Une telle analyse semble particulièrement fructueuse parce que le temps et l'espace ont été étudiés selon deux points de vues différents et pourtant complémentaires. L'étude du temps s'est focalisée principalement sur les moyens linguistiques permettant d'exprimer celui-ci dans les textes (p. ex. les adverbiaux temporels) et sur leur impact lors de la compréhension. Par contre, la manière dont les informations spatiales sont présentées dans un texte a retenu bien peu l'attention au profit de la question de savoir si et comment la multidimensionnalité de l'espace peut être reconstruite par le lecteur sur la base de la linéarité d'un texte. Un des objectifs spécifiques de ce travail est de proposer, sur la base de cette confrontation, de nouvelles hypothèses de recherches à propos du rôle des adverbiaux spatiaux lors de la construction du modèle situationnel. Un second objectif, tout aussi important, vise à déterminer comment les conclusions issues des recherches empiriques à propos de l'espace et du temps s'accordent avec les deux principales théories décrivant les processus cognitifs qui sous-tendent la compréhension : la conception constructiviste selon laquelle le lecteur cherche activement à construire un modèle situationnel et la conception mémorielle qui met l'accent sur les processus automatiques d'activation des connaissances à la source de ce même modèle.

For more than twenty years, one of the major challenges faced by psycholinguistics has been to describe the nature of the mental representation a reader builds. This question has been studied for so many years because, contrary to other processes like lexical access or syntactic analysis, the comprehension of a text was long considered to be difficult to study scientifically as its definitions were not sufficiently rigorous. Today this is no longer the case. Researchers agree to define the comprehension of a text as the construction of a situation model which combines the propositions extracted from the text with those reactivated in long-term memory. This situation model is a representation of what the text is about, not of the text itself. Among the multiple dimensions supposedly represented in the situation model, space and time has aroused the most interest. The main objective of this paper is to confront our knowledge concerning the role of these two dimensions during the construction of the situation model in order to propose new hypotheses regarding the impact of spatial adverbials on this process. A second and equally important objective is determining how the conclusions resulting from empirical research about space and time fit in with the two main views describing the cognitive processes underlying comprehension: the constructivist and the memory-based views.

\section{INDEX}

Mots-clés : compréhension de textes, conception constructiviste et mémorielle, espace, expressions adverbiales, modèle situationnel, temps

Keywords : adverbials, constructivist and memory-based view, situation model, space, text comprehension, time 


\section{AUTEUR}

\section{YVES BESTGEN}

Chercheur qualifié du F.S.R-FNRS

UCL/PSP PSOR/CECL

Place du Cardinal Mercier 10 B-1348 Louvain-la-Neuve Belgium yves.bestgen@psp.ucl.ac.be 